\title{
On the Reclassification of Short GRBs
}

\author{
Zhibin Zhang ${ }^{1}$, Yongfeng Huang ${ }^{2}$ and Hongchao Liu ${ }^{1}$ \\ ${ }^{1}$ Department of Physics, College of Sciences, Guizhou University, \\ Guiyang 550025, China; \\ email: sci.zbzhang@gzu.edu.cn \\ ${ }^{2}$ Department of Astronomy, Nanjing University, \\ Nanjing 210093, China \\ email: hyf@nju.edu.cn
}

\begin{abstract}
By collecting 17 short gamma-ray bursts with necessary data, we find a correlation of $L_{p} \propto E_{p, i}^{1.7}$, which is very consistent with that derived from a greatly expanded sample of 148 Swift long gamma-ray bursts. It is argued that the radiation mechanism of both long and short gamma-ray bursts should be similar, i.e., of quasi-thermal origin caused by the photosphere and the dissipation occurring very near the central engine. In addition, we suggest that the $E_{p, i}-L_{p}$ relation can be used to identified a burst among normal short bursts, short bursts with extended emission and long bursts with short-hard properties. We also find the ratio of peak energy to fluence in the prompt $\gamma$-ray band is a prospective discriminator, similar to the traditional duration time.
\end{abstract}

Keywords. Gamma-ray burst: general, Radiation mechanisms: general, Methods: statistical

\section{Introduction}

A correlation between the peak luminosity and the intrinsic peak energy has been found by Yonetoku et al. (2004) as $L_{p} \propto E_{p, i}^{2}$ for 11 pre-Swift long gamma-ray bursts (see also Wei \& Gao 2003; Schaefer 2003). Many efforts have been put on the physical origin and implications of the intriguing luminosity relation. For example, Lloyd-Ronning \& Ramirez-Ruiz (2002) found that bursts with highly variable curves generally have greater spectral peak energies. Since the light curve variability may be correlated with luminosity, it is then reasonable that $E_{p, i}$ and $L_{p}$ should be correlated.

Since 2005, statistical investigations of the $E_{p, i}-L_{p}$ relation of short-hard GRBs (SGRBs) become possible because of the accumulation of SGRBs with measured redshifts. Ghirlanda, Ghisellini \& Nava (2011) found that the $E_{p, i}-L_{p}$ relation also existed within individual bursts and that the short burst GRB 090510 was marginally located at the end of long GRB population, which was argued to be the evidence supporting the intrinsic origin of this relation. Furthermore, some recent works qualitatively draw a conclusion that short and long bursts should follow the same $E_{p, i}-L_{p}$ relation (Ghirlanda et al. 2009; Zhang et al. 2009; Zhang et al. 2012).

Here, we show that the $E_{p, i}-L_{p}$ relation of SGRBs does exist quantitatively (Zhang, Chen \& Huang 2012) and could be useful to discriminate different short GRBs. Moreover, we compare our relation with the recently found $E_{p, o}-$ Fluence ratio discriminator (Goldstein, Preece \& Briggs 2010) for short bursts.

\section{Results}

Among the 17 bursts in our sample (sample 1), two special "short" bursts (GRB 071020 and GRB 080913A) last longer than 2 seconds in duration but behave spectral features of 


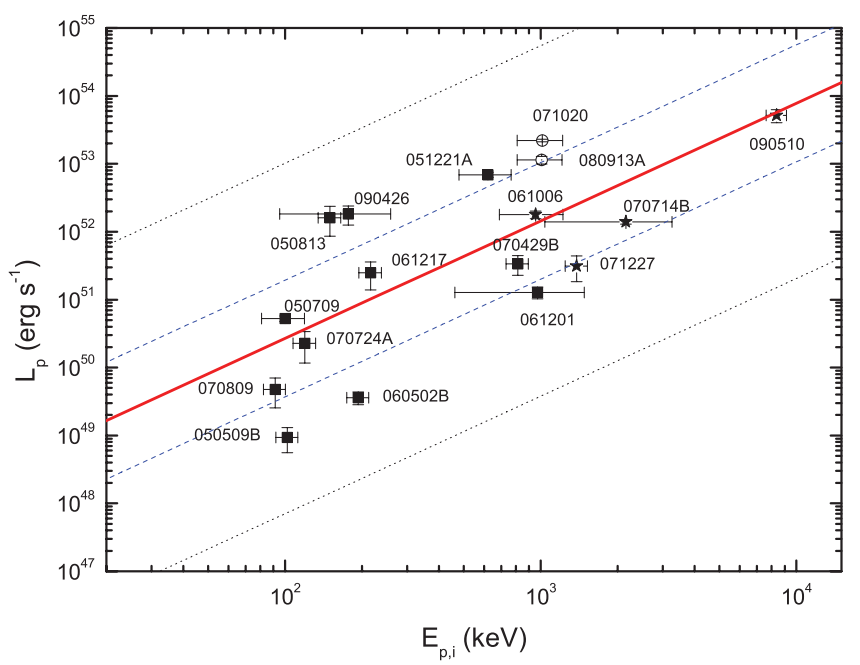

Figure 1. Rest frame peak energy versus peak luminosity for sample 1 . The solid line is the best fit to the observed data in the logarithmic frame of axes. The dashed lines and the dotted lines correspond to its $1 \sigma$ and $3 \sigma$ scatters $(\sigma=0.86)$, respectively. "Short" GRBs with EE are symbolized with dark filled stars and "long" bursts with short-hard properties are marked with open circles.

typical long bursts in both the prompt and afterglow phase. In addition, four bursts (i.e., GRB 061006, GRB 070714B, GRB 071227 and GRB 090510) with the extended emission (EE) component are included in the sample. We have also collected 148 long GRBs (110 Swift/BAT bursts, 10 Fermi/GBM bursts and 28 Konus-wind bursts) with the necessary quantities measured to construct a combined sample (sample 2) for getting the updated $E_{p, i}-L_{p}$ relation. For the samples 1 and 2 , we have $L_{p} \propto E_{p, i}^{1.7}$ with linear correlation coefficient of $R \geqslant 0.7$ and chance possibility of $P \leqslant 0.001$, which offers an ideal evidence that short and long bursts are dominated by the same radiation mechanism, that is the thermal emission (Zhang, Chen \& Huang 2012). Our newly discovered $E_{p, i}-L_{p}$ relation for sample 1 is displayed in Fig. 1, in which one can note there are three populations in the plane of $E_{p, i}$ versus $L_{p}$. Short bursts with EE reside at the high end of their $E_{p, i}-L_{p}$ relation, while "long" bursts with short-hard features seem to favor the $E_{p, i}-L_{p}$ relation of long bursts. This implies that the $E_{p, i}-L_{p}$ relation might be used to identify different kinds of SGRBs physically.

Goldstein, Preece \& Briggs (2010) find that the ratio of peak energy $\left(E_{p, o}\right)$ to energy fluence of GRBs from BATSE 5B catalog is a good discriminator used to divide all GRBs into two different classes, corresponding to short-hard and long-soft sources. From their Figures 1 and 2 we see the fraction of SGRBs is comparable to that derived from the duration $\left(T_{90}\right)$ distribution. Meanwhile, short and long bursts have larger and smaller energy ratio values $\left(E_{p, o} /\right.$ Fluence $)$, respectively. To compare this classification criterion with that based on $T_{90}$ for 17 SGRBs of sample 1, we make a plot of $T_{90}$ versus $E_{p, o} /$ Fluence in Fig. 2, where $T_{90}$ and $E_{p, o} /$ Fluence are inversely correlated with Spearman rank-order correlation coefficient of -0.53 and chance probability of $P \sim 0.02$, which indicates that SGRBs can be reclassified according to the value of $E_{p, o} /$ Fluence. Again, we can find the two "long" bursts with short-hard properties are located at the edge of normal SGRBs, near to the region of long GRBs. A Kolmogorov-Smirnov test between normal SGRBs and SGRBs with EE returns the statistic $D=0.45$ with $P \sim 0.36$, demonstrating both kinds of SGRBs are most probably drawn from distinct parent distribution, as shown in 


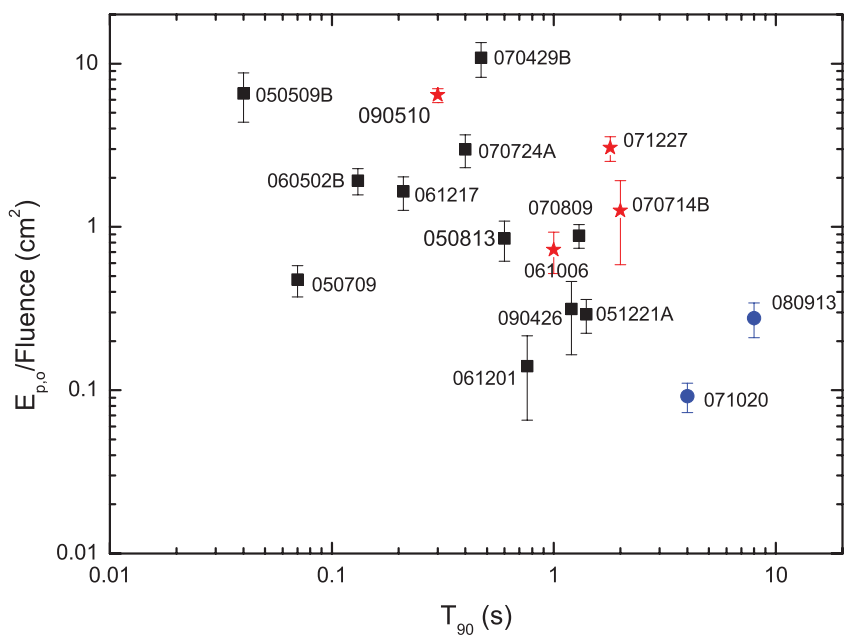

Figure 2. The energy ratio value versus energy fluence for sample 1. Normal SGRBs are symbolized with filled squares. "Short" GRBs with EE and "long" bursts with short-hard properties are marked with red filled stars and blue filled circles, respectively.

Fig. 1. Therefore, we conclude that the $E_{p, o}$-Fluence relation and the $E_{p, i}-L_{p}$ relation are two potential indicators to reclassify SGRBs in a sense of physics.

\section{Acknowledgements}

This work is supported by the National Natural Science Foundation of China (Grant No. 10943006, 11263002), Guizhou natural and scientific foundations (Grant No. 20092662, 20104014, 20117006 and 20090130) and foundations of Ministry of Education of the PRC (No. 210197, 20101174). Y.F.H. was supported by the National Natural Science Foundation of China (Grant No. 11033002) and the National Basic Research Program of China (973 Program, Grant No. 2009CB824800).

\section{References}

Ghirlanda, G., Nava, L., Ghisellini, G., et al. 2009, A\&A, 496, 585

Ghirlanda, G., Ghisellini, G., \& Nava, L. 2011, MNRAS, 418, L109

Goldstein, A., Preece, R. D., \& Briggs, M. 2010, ApJ, 721, 1329

Lloyd-Ronning, N. M. \& Ramirez-Ruiz, E. 2002, ApJ, 576, 101

Schaefer, B. E. 2003, ApJ, 583, L71

Wei, D. M. \& Gao, W. H. 2003, MNRAS, 345, 743

Yonetoku, D., Murakami, T., Nakamura, T., et al. 2004, ApJ, 609, 935

Zhang, B., Zhang, B. B., Virgili, F. J., et al. 2009, ApJ, 703, 1696

Zhang, F. W., Shao, L., Yan, J. Z., \& Wei, D. M. 2012, ApJ, 750, 88

Zhang, Z. B., Chen, D. Y., \& Huang, Y. F. 2012, ApJ, 755, 55 